\title{
Low-cost semirigid ureteroscopy is effective for ureteral stones: Experience of a single high volume center
}

\author{
Roberto Giulianelli, Barbara Cristina Gentile, Giorgio Vincenti, Luca Mavilla, Luca Albanesi, \\ Francesco Attisani, Gabriella Mirabile, Francesco Pisanti, Manlio Schettini \\ Division of Urology, Nuova Villa Claudia, Rome, Italy.
}

\begin{abstract}
Summary Aim of the study: To demonstrate how, in a center with a large number of patients, as our center is, it is possible to perform ureterolithotripsy using a limited set of instruments. Methods: We evaluated medical charts of our center related to semirigid ureteral ureteroscopy (URS) with
\end{abstract} ureterolithotripsy using Holmium laser performed from July 2004 to July 2011. Overall, 658 URS for ureteral stones were performed in 601 patients, of which 204 in proximal ureter (31\%), 86 in the mid (13.06\%) and $368(57.76 \%)$ in the distal ureter. In 504 patients (76.5\%) ureterohydronephrosis (Grade II-III) was observed. In 57 patients (8.6\%), we performed a bilateral approach at the same time, but most patients had a solitary distal ureteral stone. 106 patients $(16.1 \%)$ had more than one stone in their distal ureter and $96(14.8 \%)$ had a proximal ureteral stone treated in the same surgery as well.

Results: The overall stone-free rate for ureteral stones was $86.1 \%$ (567/658). Success rates for proximal, medial and distal ureteral stones were $68.13 \%$ (139/204 patients), $84.8 \%$ (73/86 patients) and 96.4\% (355/368 patients), respectively. One hundred and twenty patients (18.3\%) required additional surgical treatment for their stones beyond the initial URS, including a second URS in 97 patients (14.74\%) and URS plus Retrograde Intra-Renal Surgery (RIRS) in 23 patients (3.54\%). The overall stonefree rate after the second treatment was $99.3 \%$. Intra-operative complications accounted for $5.92 \%$ and consisted of ureteral perforations in 16 pts (2.4\%), erosions of urothelium leading to significant bleeding in 15 pts $(2.27 \%)$, severe pain in 4 pts $(0.6 \%)$, fever in 3 pts $(0.45 \%)$ and one case of ureteral avulsion $(0.15 \%)$.

Conclusions: This study demonstrates that the use of Holmium laser lithotripsy is a safe and effective means of treating ureteral stones regardless of sex, age, stone location, or stone size. The instrumentation we used was extremely limited, in order to reduce costs related to the procedure to an absolute minimum whilst maintaining the two quality indicators for the procedure, namely successrate and length of hospitalisation (86.1\% and 34 hours).

KEY WORDS: Ureteral calculi; Ureteroscopy; Holmium laser; Lithotripsy.

Submitted 25 February 2014; Accepted 19 May 2014

No conflict of interest declared.

\section{INTRODUCTION}

Urolithiasis is a common and costly disease displaying increasing worldwide prevalence and incidence rates (1). The lifetime risk of urolithiasis is estimat-ed to be between 5\% and 12\% in Europe and USA, afflicting 13\% of men and $7 \%$ of woman (2). Extracorporeal shock wave lithotripsy (ESWL) remains the recommended first-line treatment for most stones (3) but for those stones lodged in the ureter, ureteroscopy (URS) has become the most common treatment method (4). URS is the most commonly advocated treatment for patients with ureteral calculi with a stone-free rate higher than $90 \%$ after a single treatment. Open ureterolithotomy is no longer considered as a valid op-tion in a well equipped endourological center. With the introduction of in situ extracorporeal shock wave lithotripsy and different intracorporeal techniques in urology practice, up to $95 \%$ of ureteral stones can be successfully treated with a minimally invasive method (5-10). In 2007 the EAU Guideline recom-mended that for ureteral stones requiring removal, because up to $98 \%$ of ure-teral calculi $<5 \mathrm{~mm}$ in diameter are likely to pass spontaneously, both SWL and URS are acceptable first-line treatments in healthy non-pregnant adults who have unilateral calculi (2). In this meta-analysis, overall stone-free rates for SWL and URS differed, depending on stone size, location, and treatment method. Technological progress in the field of semirigid ureteroscope tech-nology, chiefly involving the miniaturization of the scopes and improved durability, and the introduction of the Holmium:YAG laser, with its precise and powerful thermal decomposition mechanism, its excellent safety profile and the ability of laser energy to be delivered through small flexible fibers, have opened up the path for fragmentation of stones of all composition types (5-10). Use of this increasingly highperformance instrumentation (11), along with the use of dedicated devices which promote access to the ureter or prevent ret-ropulsion of the kidney stone (12), have considerably increased the costs of these procedures. The aim of this study was to demonstrate how, in an high volume center, such our center is,it is possible to perform a ureterolithotripsy for ureteral stones using a minimum set of instruments to complete the treat-ment. 


\section{Materials AND METHOdS}

We evaluated medical charts of our centre related to semirigid ureteral ureteroscopy (URS) with ureterolithotripsy using Holmium laser performed, from July 2004 to July 2011, by four urologists. Of these, two performed more than 200 upper tract endourological procedures, one almost 100, and one less than 50. In total they performed 658 URS in 601 patients, 457 males and 144 females, with an average age of 43.5 years (15-72 years). Stones were in the proximal ureter in 204 patients $(31 \%)$, in the mid ureter in $86(13.06 \%)$ and in the distal ureter in 368 $(57.76 \%$ ). The average stone diameter was $9.1 \mathrm{~mm}$ (range 3-22). Most patients had a solitary distal ureteral stone, but $106(16.1 \%)$ had multiple stones in the distal ureter and $96(14.8 \%)$ were treated also for proximal ureteral stones whilst undergoing the same surgery. In 57 patients (8.6\%) URS was bilaterally performed and in 37 (5.6\%) the procedures were performed under emergency conditions due to acute renal failure.

URS was offered in cases where the stone failed a trial of passage for up to 10-12 days from the start of expulsive therapy. Preoperative work-up consisted of Renal Ultrasound (RUS) in 645 patients (98.02\%), Plain KidneyUreter-Bladder X-ray (KUB) in 453 patients (68.8\%) and Computerized Tomography (TC) in 67 patients (10.1\%). In 504 patients $(76.5 \%)$ a ureterohydronephrosis was observed (grade II-III), in 534 patients (81.5\%) drugresistant pain, in 64 patients (9.7\%) haematuria, and in 65 patients (9.87\%) fever. All patients were hospitalised on the same day on which we carried out the endoscopic procedure, 602 of those (92.4\%) were performed under general anaesthesia. Only 56 patients (8.5\%), due to severe chronic obstructive pulmonary disease (COPD), were given an epidural anaesthesia.

Our technique involved use of a Storz semirigid ureteroscope to make an initial inspection of the bladder to rule out the simultaneous presence of other diseases (e.g. malignancies, flat bladder lesions), and to identify the ureteral orifice to be approached. Having assessed its characteristics, and in particular its shape, orifice was cannulated using a $4 \mathrm{~F}$ open-toe ureteral catheter advanced through the working channel of the ureteroscope up to about 3-4 $\mathrm{cm}$ from the orifice, acting as a "working wire". At this point, using the pressure of the irrigation flow and relying on the catheter, the affected ureter was reached with an initial movement which involved a delicate lifting of the instrument with subsequent abduction. We proceeded with extreme caution along the ureter, minimizing the flow of water washing, to reduce the risk of pushing the stone upwards, thus reaching the stone and then proceeding with the ureterolithotripsy using the Holmium laser with an 0.8 to $1 \mathrm{~J}$ energy pulse and 8 to $10 \mathrm{~Hz}$ frequency. The stone was then fragmented with the laser until all pieces were approximately 2 $\mathrm{mm}$ or smaller. Stone fragments were not routinely extracted and the decision to place a ureteral stent upon completion of the procedure was based on preoperative grade of ureterohydronephrosis and intra-operative findings. We positioned a JJ stent in all patients with severe ureterohydronephrosis or when other conditions rendered it necessary (i.e., ureteral wall injury, bleeding, severe inflammatory reaction against the ureteral wall).
On the other hand, in 214 patients we left in place the ureteral catheter previously used as a guide, fixed by its distal end to a Foley catheter, positioned at the end of the procedure for 24 hours. We did not use a basket to prevent the stone from being pushed upwards. Fluoroscopy was not requested for treatment of distal ureteral stones, when calculi were positioned below the intersection with the iliac vessels, whatever their diameters. We used fluoroscopy in 18 patients $(2.73 \%)$ including 14 with stones $(2.12 \%)$ in the proximal ureter and $5(0.75 \%)$ in the mid ureter. Some patients had concomitant renal calculi that were deemed to be clinically insignificant (usually $<3$ $\mathrm{mm}$ ) and were not treated at the time of URS. Patients were instructed to strain their urine postoperatively and to bring the retrieved fragments to their next outpatient appointment so they could be sent for analysis.

The charts, including operative reports, were reviewed and data concerning patient and stone characteristics, duration of surgery, use of stents and use of secondary procedures were collected. We recorded data concerning body mass index (BMI) and gender. We also reviewed imaging studies of the patients at 30-day postoperative follow up to assess stone-free rates. Computed tomography (CT), renal ultrasound (US) and plain abdominal radiography (KUB) were used for imaging. We compared preand postoperative imaging to determine whether renal stones seen on postoperative films were consistent with pre-existing, untreated renal stones or with new, proximally migrated fragments of a ureteral stone. Data concerning complications was also recorded. Statistical analyses were performed using Student $t$ test and chisquare analysis.

\section{RESULTS}

Overall, 658 URS for ureteral stones were performed in 601 patients, of which 204 in proximal ureter (31\%), 86 in the mid (13.06\%) and $368(57.76 \%)$ in the distal ureter. In 504 patients (76.5\%) ureterohydronephrosis (Grade II-III) was observed. In 57 patients (8.6\%), we performed a bilateral approach at the same time, but most patients had a solitary distal ureteral stone. 106 patients (16.1\%) had more than one stone in their distal ureter and $96(14.8 \%)$ had a proximal ureteral stone treated in the same surgery as well.

The overall stone-free rate for ureteral stones was $86.1 \%$ (567/658). Success rates for proximal, medial and distal ureteral stones were $68.13 \%$ (139/204 patients), 84.8\% (73/86 patients) and 96.4\% (355/368 patients), respectively. One hundred and twenty patients (18.3\%) required additional surgical treatment for their stones beyond the initial URS, including a second URS in 97 patients (14.74\%) and URS plus Retrograde Intra-Renal Surgery (RIRS) in 23 patients (3.54\%). The overall stonefree rate after the second treatment was $99.3 \%$. Four patients $(0.6 \%)$ were judged to be failures (i.e. not stonefree) in that they had new renal stones postoperatively, consistent with proximal migration of ureteral stones after fragmentation that did not clear up. These fragments, however, were $2 \mathrm{~mm}$ in size in $75 \%$ of patients; in one patient, a $5 \mathrm{~mm}$ fragment was found in the ureter by postoperative imaging but the patient was subsequently lost 
in the follow-up, and was therefore classified as a failure. The overall stone-free rate for the treatment of ureteral stones was worse in the obese group than in the nonobese group, but not statistically significant ( $82 \%$ vs $76 \%$, $\mathrm{P}=\mathrm{NS})$. The mean operating time was 32 minutes (1252 minutes, depending on stone burden and impaction) and mean hospital stay was 34 hours (26-42 h).

Two hundred and twelve patients (32.2\%) had a ureteral stent placed before their URS. We performed a bilateral approach in 57 patients (8.6\%), and of these the procedure was performed under emergency conditions of acute renal failure in 37 patients (5.6\%). Fifty-three patients $(8.05 \%)$ had concomitant urinary tract infection, 69 (10.4\%) had severe ureterohydronephrosis (III grade), 11 (1.94\%) ureteral wall injury, 9 (1.36\%) bleeding and $13(1.97 \%)$ a severe inflammatory response of the ureteral wall. In all other cases we left the 4 or $5 \mathrm{~F}$ ureteral catheter used during the procedure after completely removing all of the ureteral fragments over $3 \mathrm{~mm}$, leaving it out of the external urethral meatus where it was attached to the Foley catheter.

Complications were uncommon. Intra-operative complications accounted for $5.92 \%$ and consisted of ureteral perforations in 16 pts $(2.4 \%)$, erosions of urothelium leading to significant bleeding in 15 pts $(2.27 \%)$, severe pain in 4 pts $(0.6 \%)$, fever in 3 pts $(0.45 \%)$ and one case of ureteral avulsion $(0.15 \%)$. Conversion to open surgery was carried out in one patient $(0.15 \%)$ with a distal ureter stone associated with a neoplasm of the upper tract, where the endoscopic ureteral avulsion manoeuvre occurred accidentally. There was no immediate postoperative mortality. Fifty-three patients $(8.05 \%)$ presented a postoperative urinary tract infection, none of whom required hospitalisation.

Late complications were persistent haematuria in 66 patients (10.03\%) and severe dysuria in 146 patients (25.2\%). In 87 patients with dysuria (13.25\%) long term use of NSAIDs was required. Early stent removal (within 30 days from surgery) was necessary in 116 patients (17.6\%) whereas 98 patients $(14.8 \%)$ presented at the emergency department complaining of pain in the side of the procedure which was related to the stent, but did not require hospitalisation.

\section{Discussion}

The management of ureteral stones has seen a change from open surgery to SWL and to endoscopic and laparoscopic surgery. Intracorporeal lithotripsy devices and URS have made treatment of ureteric stones much more convenient. Rigid URS was first applied for the treatment of distal ureteral calculi in the 1980s. Although large (> 10F) diameter ureteroscopes were used, success rates of $>90 \%$ were achieved (13). With the development of smaller caliber ureteroscopes and the introduction of improved instrumentation, including the Holmium:YAG laser, ureteroscopy has evolved into a safer and more effective method of treating ureteral stones.

In our study, the overall stone-free rate of URS was comparable to other studies, with stone-free rates ranging from $75 \%$ to $93 \%$ (14). In fact we obtained an $86.1 \%$ overall stone-free rates after Holmium laser ureterolithotripsy.
Leijte JA et al. (15) showed in 105 ureteroscopic Holmium laser lithotripsies procedures a total success rate of $84.8 \%$ and Ullah et al. in 88 ureteral stone cases treated with ureteroscopic Holmium laser lithotripsy reported an overall success rate with satisfactory fragmentation in $85.15 \%$ (16). Safwat et al. observed 239 patients (199 males and 40 females) with an average stone burden of $9.8 \mathrm{~mm}$ (range 4 to $20 \mathrm{~mm}$ ) and reported a success rate of $96.3 \%$ after a single session which increased to $99 \%$ after 2 sessions (17).

In our experience, we have obviously observed different results in terms of stone-free rates, according to the site in which the stone was located. In fact, success rates for proximal, mid and distal ureteral stones were $68.13 \%$, $84.8 \%$ and $96.4 \%$,respectively. Similarly,in a total of 88 stones, 22 (25\%) in the upper ureter, 24 (27.27\%) in the middle ureter and $42(47.72 \%)$ in the lower ureter, Ullah et al. (17) showed success rates of $72.72 \%, 87.5 \%$ and $95.23 \%$, respectively.

Similar results were also obtained by Subhani et al. (18), who carried out ureteroscopic Holmium laser lithotripsy in 209 patients with stones in the lower ureter, 266 in the middle ureter and 65 in the upper tract of the ureter, achieving success rates of $94.73 \%, 95.11 \%$ and $44.61 \%$, respectively.

Use of flexible ureteroscopy for treating stones located in the proximal tract, would have propably improved our results in terms of stone-free rates in line with available data (68.13\% vs 93.1\%) (19). However, owing to the costs in-volved, use of this method in our center is exclusively limited to the treatment of complex calculi or for the treatment of intrarenal lithiasis $>2 \mathrm{~cm}$.

In our experience, one hundred and twenty patients (18.3\%) required addi-tional surgical treatment for their stones beyond the initial URS to achieve an overall stonefree rate of $99.3 \%$. These results, including a second URS in 97 pts (14.74\%) and URS plus RIRS in 23 pts (3.54\%). The Holmium laser pro-vides the gold standard for intracorporeal lithotripsy (20) and its use proved to be crucial in our experience.

Holmium laser can effectively fragment any stone regardless of composition or size and can reach the entire urinary tract since it can be deployed on rigid and flexible ureteroscopes. Furthermore, compared to other intracorporeal lithotripsy, Holmium laser yields the smallest fragment size, with many even smaller than $1 \mathrm{~mm}$ (20).

According to international experiences, the use of the Holmium laser, enabled us to attain complete pulverization of the stones at the end of our procedures $(21,22)$. This reduced risk of complications to a minimum (none steinstrasse) and reduced the risk of retropulsion of the stone to a minimum, whereas figures are undoubtedly higher when ballistic lithotripsy is used (23).

Bapat et al. compared the success rates of Lithoclast and Holmium laser-assisted ureterorenoscopy in 394 patients assessing at 2 weeks fragmentation into fine pieces of stones and their passage. This occurred in 166/193 (86.01\%) patients in the Lithoclast group and in 195/201 (97.01\%) in the laser group (24).

In general complications are uncommon. In Bapat's experience, the complications and the need for auxiliary procedures were significantly less for Holmium laser-assisted 
ureteroscopy when compared with pneumatic lithotripsy (24). Ullah et al. reported an overall complication rate of $17.04 \%$; the main complications included ureteral perforation $(n=2)$, ureteral avulsion $(n=1)$, urosepsis $(n=2)$ and stone migration $(\mathrm{n}=10)(17)$. Subhani et al. described an overall complication rate of $11.83 \%$. The main complications included mucosal lacerations (9.25\%), perforation $(2.40 \%)$, ureteric avulsion (0.18\%) (18).

In our experience, intra-operative complications accounted for $5.92 \%$ of our cases and consisted of ureteral perforations in 16 patients (2.4\%) and erosions of urothelium leading to significant bleeding in 15 patients (2.27\%). In one patient which presented with a distal ureter stone associated with a neoplasm of the upper tract, we had to convert the endoscopic procedure to open surgery $(0.15 \%)$, because the endoscopic manoeuvre accidentally caused ureteral avulsion. Late complications included persistent haematuria in 66 patients (10.03\%) and severe dysuria in $146(25.2 \%)$. Out of them 87 patients required long term NSAIDs treatment (13.25\%).

\section{Costs}

Conducting a stone-free Holmium laser-assisted ureteroscopy is less costly than a SWL procedure (higher number of treatments required to obtain stone-free condition with SWL, higher risk of steinstrasse after SWL) (25). A study reported the cost of stone removal for both the upper and lower ureter using SWL significantly higher compared to endoscopic procedures (ureteroscopy with semirigid ureteroscope and the use of pneumatic lithoclast, or ureteroscopy with flexible ureteroscope and the use of Holmium YAG Laser).

The median cost for the upper ureter was $€ 828$ vs $€$ 474.50 and $€ 396$ respectively, and for the lower ureter, $€ 826$ vs $€ 396$ and $€ 271, p<0.001$ (26). Esuvaranathan et al. observed, in a prospective consecutive series of 64 patients who underwent transurethral laser ureterolithotripsy using a $7.2 \mathrm{~F}$ semirigid ureteroscope, that the 3 -year cost-benefit analysis revealed a smaller difference in cost than expected and the 5-year analysis was advantageous for laser lithotripsy because of its higher success rate (27).

At present, costs constitute an increasingly important problem and, unfortunately, they have an increasing influence on the treatment choices in the clinical setting. All this has in fact prompted us to gradually reduce our instrumentation to a minimum and to choose the semirigid ureteroscope as a first choice instead of the flexible one. The latter is instead used only for treating complex ureteral calculi. We have gradually developed a technique for accessing the ureter which requires the use of a simple ureteral catheter, avoiding the use of additional guide wires or instruments to dilate the ureteral ostium. We rely on a simple manoeuvre exploiting the pressure of the washing fluid and the presence of the catheter. The affected ureter is reached with an initial movement which involves a delicate lifting of the instrument and its subsequent abduction. The extreme attention and care with which the movement is carried out reduces the risk of lesions to the ureter and/or its intussusception to a minimum. In our experience, intra-operative complications accounted for $5.92 \%$ and we performed conversion to open surgery in only one patient $(0.15 \%)$. Needless to say, we are in complete agreement with all those that sustain that the experience of the surgeon is of the utmost importanc (15) and plays a crucial role in determining a reduced rate of method-related complications. We also feel it is important to have procedure-dedicated surgeons who exclusively carry out all these procedures.

In our experience, the mean hospital stay was 34 hours (26-42 h), whilst Rombi et al. observed a hospital stay for upper ureter cases of 2.48 days, and 2.43 days for lower ureter, respectively (26).

\section{Conclusions}

This study demonstrates that the use of Holmium laser lithotripsy is a safe and effective means of treating ureteral stones regardless of sex, age, stone location, or stone size. We found that the overall stone-free rates before Holmium laser ureterolithotripsy were $86.1 \%$ and $99.3 \%$ after the first and second treatment, respectively.

Complications were uncommon. The instrumentation we used was extremely limited, in order to reduce costs related to the procedure to an absolute minimum whilst maintaining the two quality indicators for the procedure, namely success-rate and length of hospitalisation (86.1\% and 34 hours), which reflect those obtained with other experiences.

\section{REFERENCES}

1. Hesse A, Brandle E, Wilbert D, et al. Study on the prevalence and incidence of urolithiasis in germany comparing the years 1979 vs 2000. Eur Urol. 2003; 44:709-13.

2. Preminger GM, Tiselius HG, Assimos DG, et al.: Guideline for the management of ureteral calculi. J.Urol 2007; 178:2418-34.

3. Osman MM, Alfano Y, Kamp S, et al. 5-years follow up od patients with clinically insignificant residual fragmenta and after extracorporeal shock wave lithotripsy. Eur Urol. 2005; 47:860-4.

4. Bader MJ, Eisner B, Porpiglia F, et al. Contemporary management of ureteral stones. Eur Urol. 2012; 61:764-771.

5. Yinghao S, Linhui W, Songxi Q, et al. Swiss lithoclast pneumatic lithotripter: report of 150 cases. J Endourol. 2000; 14:281-3.

6. Ather MH, Paryani J, Memon A, Sulaiman MN. A 10-year experience of managing ureteric calculi: Changing trends towards endourological interven-tion-is there a role of open surgery? BJU Int. 2001; 88:173-177.

7. Dirim A, Tekin MI, Aytekin C, et al. Ureteroscopic treatment of proximal ureter stones with aid of an antegrade occlusion balloon catheter. Acta Radiol. 2006; 47:103-6.

8. Kupeli B, Biri H, Isen K, et al. Treatment of ureteral stones: comparison of extracorporeal shock wave litho-tripsy and endourologic alternatives. Eur Urol. 1998; 34:474-479.

9. Naqui SA, khalogi M, Zafar MN, Rizwi SA. Treatment of ureteric stones. Comparison of laser and pneumatic lithotripsy. Br J Urol. 1994; 74:694-698.

10. Maislos SD, Volpe M, Albert PS, Raboy A. Efficacy of the stone cone for treatment of proximal ureteral stones. J Endourol. 2004; 18:862-4.

11. Sofer M, Watterson JD, Wollin TA, Nott et al.: Holmium:YAG 
laser lithotripsy for upper urinary tract calculi in 598 patients. J Urol. 2003; 44:482-6.

12. Kijvikai K, Haleblian GE, Preminger GM, de la Rosette J. Shock wave lithotripsy or ureteroscopy for the management of proximal ureteral calculi: an olddiscussion revisite. J Urol. 2007; 178:1157-63.

13. Tawfiek ER, Bagley DH.: Management of upper urinary tract calculi with ureteroscopic techniques. Urology. 1999; 53:25-31.

14. Park H, Park M, Park T. Two-year experience with ureteral stones: extracorporeal shockwave lithotripsy $v$ ureteroscopic manipulation. J Endourol. 1998; 12:501-504.

15. Leijte JA, Oddens JR, Lock TM. Holmium laser lithotripsy for ureteral calculi: predictive factors for complications and success. $J$ Endourol. 2008; 22:257-60.

16. Ullah I, Wazir BG, Alam K, et al. Evaluation of safe-ty and efficacy of ureteroscopic lithotripsy in managing ureteral calculi. Ann Pak Inst Med Sci. 2011; 7:119-112.

17. Safwat AS, Bissada NK, Kumar U, et al. A multi-institutional study demonstrating the safety and effica-cy of Holmium laser ureterolithotripsy. UroToday Int J. 2012; 5:349.

18. Subhani GM, Javed SA, Iqbal Z, et al. Outcome of Retrograde Ureteroscopy for the Management of Ureteric Calculi: Four Years Experience. A.P.M.C. 2009; 3;8-12.

19. Cocuzza M, Colombo JRJr, Cocuzza AL, et al. Outcomes of flexible ureteroscopic lithotripsy with holmium laser for upper urinary tract calculi. Int Braz J Urol. 2008; 34:143-9.
20. Preminger GM, Tiselius HG, Assimos DG, et al. Guideline for the management of ureteral calculi. J Urol 2007; 178:2418-34.

21. Jong-Hyun Lee, Seung Hyo Woo, Eun Tak Kim, Dae Kyung Kim, Jinsung Park Comparison of patient satisfaction with treatment outcomes between ureteroscopy and Shock Wave Lithotripsy for proximal ureteral stones Korean J Urol. 2010; 51:788-793.

22. Wu CF, Shee JJ, Lin WY, et al. Comparison between extracorporeal shock wave lithotripsy and semirigid ureterorenoscope with holmium:YAG laser lithotripsy for treating large proximal ureteral stones. J Urol. 2004; 172:1899.

23. Teichman JM1, Vassar GJ, Bishoff JT, Bellman GC Holmium:YAG lithotripsy yields smaller fragments than lithoclast, pulsed dye laser or electrohydraulic lithotripsy. J Urol. 1998; 159:17-23.

24. Bapat SS, Pai KV, Purnapatre SS, et al. Comparison of holmium laser and pneumatic lithotripsy in managing upper-ureteral stones. J Endourol. 2007; 21:1425-7.

25. Nabi G, Downey P, Keeley F, et al. Extracorporeal shock wave lithotripsy (ESWL) versus ureteroscopic management of ureteric calculi. Cochrane Database Syst Rev. 2007; CD006029.

26. Rombi T, Triantafyllidis A, Fotas A, et al. Socioeconomic evaluation of the treatment of ureteral lithiasis. Hippokratia. 2011; 15:252-7.

27. Esuvaranathan K, Tan EC, Tan PK, Tung KH. Does transurethral laser ureterolithotripsy justify its cost? J Urol. 1992; 148:1091-4.

\section{Correspondence}

Roberto Giulianelli, MD (Corresponding Author)

roberto.giulianelli@virgilio.it

Barbara Cristina Gentile, MD

Giorgio Vincenti, MD

Luca Mavilla, MD

Luca Albanesi, MD

Francesco Attisani, MD

Gabriella Mirabile, MD

Francesco Pisanti, MD

Manlio Schettini, MD

Division of Urology, Nuova Villa Claudia

Via Flaminia Nuova, 280 - Rome, Italy 